\title{
Three-dimensional joint inversion of gravity and magnetic anomalies using fuzzy c-means clustering
}

\author{
Yosuke Teranishi $^{(1)}$, Hitoshi Mikada ${ }^{(1)}$, Tada-nori Goto ${ }^{(1)}$ and Junichi Takekawa ${ }^{(1)}$ \\ ${ }^{1}$ Dept. of Civil and Earth Res. Eng., Kyoto University
}

\begin{abstract}
We present a 3D joint inversion method to estimate two physical parameters, density and magnetization of subsurface materials using field intensity measurements. In the method, we introduce the fuzzy c-means (FCM) clustering technique to relate gravity with magnetic data. In the approach, the subsurface structure is discretized to a set of rectangular prisms. For estimating the density and magnetization of each prism, we minimize the quadratic norm of the residuals between the observed data and the calculated responses from inversion model. Two regularization terms, i.e. the roughness and the similarity of the two physical parameters, are introduced in our joint inversion to control the degree of model roughness and similarity. We determine their regularization parameters using the L-curve criterion. We apply our method to a numerical model which represents submarine massive sulphides (SMS). The joint inversion results, which have the advantages of both gravity and magnetic inversion, show better accuracy and resolution than the individual ones.
\end{abstract}

\section{INTRODUCTION}

Natural resources such as submarine massive sulphides (SMS), located at the shallow sub-seafloor depths, are attractive material due to the recent rapid growth of global economical activities. However, their deposits locating below deep seafloor restricts the efficient survey like the metallic mineral deposits survey on land. Furthermore, the lithology around SMS is complicated due to hydrothermal alteration, which restricts the samplings of rock cores from SMS. These necessitate detailed exploration using various kinds of geophysical surveys available near the seafloor. We, therefore, need to develop a way to multiple geophysical surveys which have sensitivities different from each other to the subsurface targets. So-called joint inversions using geostatistical method of multiple data sets have been proposed to estimate the major lithological properties of rocks (e.g., Bosch, 1999). Also, joint inversions using a cross-gradient function which measures structural similarity (Fregoso et al., 2009), or the empirical petrophysical relationships between model parameters increase the accuracy and resolution of three-dimensional (3D) geophysical models.

In general, petrophysical estimation of the relation between two different physical properties of rocks requires a fitting of empirical functions between the two properties (e.g., Jegen et al., 2009).
On the other hand, Sun et al. (2012) introduce the fuzzy c-means (FCM) clustering technique in their joint inversion of seismic traveltime and gravity data to consider the petrophysical relation between physical properties. The FCM clustering technique has an advantage that it does not necessitate any empirical equation but deals with a linear combination of the influence from multiple clusters given a piece of data to belong to plural clusters in the parameter space formed by the petrophysical parameters. Teranishi et al. (2013) also indicates the effectiveness of joint inversion of gravity and magnetic data using the FCM clustering technique.

In this paper, we incorporate model smoothness regularization with petrophysical regularization between two physical properties using the FCM clustering technique, and propose a new joint inversion method that effectively determines regularization parameters. We test our inversion method using synthetic numerical experiments for SMS, and compare the results from our joint inversion with those from an inversion scheme applied independently for every parameter.

\section{FORMULATION OF INDIVIDUAL INVERSION}

The gravity and magnetic inversions are formulated as optimization problems that minimizes the following each objective function $U_{g}, U_{m}$. 


$$
\begin{aligned}
& U_{g}=\left\|\mathbf{W}_{\mathbf{g}}\left(\mathbf{d}_{\mathbf{g}}-\mathbf{A}_{\mathbf{g}} \boldsymbol{\rho}\right)\right\|^{2}+\alpha_{g}{ }^{2}\|\mathbf{C} \boldsymbol{\rho}\|^{2} \\
& U_{m}=\left\|\mathbf{W}_{\mathbf{m}}\left(\mathbf{d}_{\mathbf{m}}-\mathbf{A}_{\mathbf{m}} \mathbf{m}\right)\right\|^{2}+\alpha_{m}{ }^{2}\|\mathbf{C} \mathbf{m}\|^{2},
\end{aligned}
$$

The vectors $\left(\mathbf{d}_{\mathrm{g}}, \mathbf{d}_{\mathbf{m}}\right)$ are gravity and magnetic anomaly vector. The matrices $\left(\mathbf{A}_{\mathbf{g}}, \mathbf{A}_{\mathbf{m}}\right)$ are sensitivity matrixes for unit density and magnetization in the $j$ th prism to the $i$ th data. $\left(\mathbf{W}_{\mathbf{g}}\right.$, $\mathbf{W}_{\mathbf{m}}$ ) are weighting matrices. If noise contaminating each data is uncorrelated each other and has a standard deviation of $\left(\sigma_{g i}, \sigma_{m i}\right)$, the weighting matrices are usually defined as $\mathbf{W}_{\mathbf{g}}=\operatorname{diag}\left\{1 / \sigma_{g l}, \ldots\right.$, $\left.1 / \sigma_{g n}\right\}, \mathbf{W}_{\mathbf{m}}=\operatorname{diag}\left\{1 / \sigma_{m l}, \ldots, 1 / \sigma_{m n}\right\}$. The notation \|\| in the above two equations is the L-2 norm measure. In each equation (1) and (2), to avoid unstableness, we add a smoothing Laplacian operator matrix $\mathbf{C}$ as a smoothness regularization term to our inversion algorithm. Smoothness regularization parameters $\alpha_{\mathrm{g}}$ and $\alpha_{\mathrm{m}}$ control a degree of spatial smoothing in the inversion.

\section{FUZZY C-MEANS CLUSTERING ALGORITHM}

FCM clustering algorithm assigns membership to each data point corresponding to each cluster center on the basis of distance between the cluster center and the data point. Mathematically, it can be expressed as the minimization of the following objective function

$$
J=\sum_{k=1}^{M} \sum_{i=1}^{C}\left(u_{k i}\right)^{q}\left\|\mathbf{x}_{\mathbf{k}}-\mathbf{v}_{\mathbf{i}}\right\|^{2},
$$

where $M$ and $C$ are the number of model prisms and clusters respsctively, $\boldsymbol{x}_{\boldsymbol{k}}$ is the $k$ th data $\left(\boldsymbol{x}_{\boldsymbol{k}}=\left[m_{k}\right.\right.$ $\left.\left.\rho_{k}\right]^{\mathrm{T}}, k=1, \ldots, M\right)$ and $\boldsymbol{v}_{\boldsymbol{i}}$ is the $i$ th cluster center $\left(\boldsymbol{v}_{\boldsymbol{i}}=\right.$ $\left.\left[m_{i}{ }^{*} \rho_{i}^{*}\right]^{\mathrm{T}}, i=1, . ., C\right)$. In this study, $\boldsymbol{x}_{\boldsymbol{k}}$ represents the value of density and magnetization in the $k$ th prism. Similarly, $\boldsymbol{v}_{\boldsymbol{i}}$ is also a vector defined by the two physical parameters in equation (3). $u_{k i}$ is the membership function that measures the degree to which the two model parameters of $k$ th prism belong to the $i$ th cluster. The parameter $q$ controls the degree of fuzziness of the resulting membership functions. In this study, we set $q=2.0$, which is widely accepted as a good choice (Hathaway and Bezdek, 2001).

\section{JOINT INVERSION WITH FCM CLUSTERING CONSTRAINTS}

Teranishi et al. (2013) formulated the joint inversion algorism by incorporateing equation (1) with (2) and considered a coupling measure based on the FCM clustering as a petrophysical similarity regularization term which combined two model parameters and they obtained the following equation;

$$
\begin{aligned}
U= & \beta^{2}\left\|\mathbf{W}_{\mathbf{g}}\left(\mathbf{d}_{\mathbf{g}}-\mathbf{A}_{\mathbf{g}} \mathbf{\rho}\right)\right\|^{2}+\left\|\mathbf{W}_{\mathbf{m}}\left(\mathbf{d}_{\mathbf{m}}-\mathbf{A}_{\mathbf{m}} \mathbf{m}\right)\right\|^{2} \\
& +\alpha_{g}{ }^{2}\|\mathbf{C} \boldsymbol{\rho}\|^{2}+\alpha_{m}{ }^{2}\|\mathbf{C} \mathbf{m}\|^{2} \\
& +\lambda^{2}\left\{\sum_{k=1}^{M} \sum_{i=1}^{C}\left(u_{k i}\right)^{q}\left\|\mathbf{x}_{\mathbf{k}}-\mathbf{v}_{\mathbf{i}}\right\|^{2}+\eta^{2} \sum_{i=1}^{C}\left\|\mathbf{v}_{\mathbf{i}}-\mathbf{t}_{\mathbf{i}}\right\|^{2}\right\} .
\end{aligned}
$$

In the equation (4), $\boldsymbol{t}_{\boldsymbol{i}}$ is a possible pair of density and magnetization which we give as a priori information from petrophysical measurements $\left(\boldsymbol{t}_{\boldsymbol{i}}=\right.$ $\left[\begin{array}{ll}\rho_{i}^{\text {ture }} & m_{i}^{\text {ture }}\end{array}\right]^{\mathrm{T}}$ ). The last term measures the distance between cluster centers updated by FCM algorithm, $\boldsymbol{v}_{i}$, and target cluster centers determined from a priori information, $\boldsymbol{t}_{\boldsymbol{i}}$. The introduction of this last term ensures the well behaved convergence of FCM algorithm, while enabling us to guide the search for cluster centers to the desired locations based on a priori petrophysical information (Sun et al., 2012).

The parameter $\beta$ adjusts the weighting ratio of gravity and magnetic in data misfit to $1: 1$. The regularization parameter $\lambda$ determines the weight of the imposed FCM clustering against the data misfit and the model smoothness. The smoothness regularization parameters $\alpha_{\mathrm{g}}$ and $\alpha_{\mathrm{m}}$ control the weight of smoothness constraint against the data misfit and the model similarity.

In the regularized inversion like the equation (4), an objective function is solved by using several regularization parameters. Then, we have to choose the optimal regularization parameter based on some kind of criterion, and model is updated by the chosen regularization parameters. In the inversion which contains some regularization terms like equation (4), the computation time of the inversion will increase rapidly, in proportion as the number of regularization parameters.

In this study, to avoid increase of inversion computation time, we do not apply smoothness regularization to model parameters, but to membership function in the FCM clustering and modified our objective function $U$ to following function;

$$
\begin{aligned}
& U=\beta^{2}\left\|\mathbf{W}_{\mathbf{g}}\left(\mathbf{d}_{\mathbf{g}}-\mathbf{A}_{\mathbf{g}} \mathbf{\rho}\right)\right\|^{2}+\left\|\mathbf{W}_{\mathbf{m}}\left(\mathbf{d}_{\mathbf{m}}-\mathbf{A}_{\mathbf{m}} \mathbf{m}\right)\right\|^{2} \\
& +\lambda^{2}\left\{\sum_{k=1}^{M} \sum_{i=1}^{C}\left(u_{k i}\right)^{q}\left\|\mathbf{x}_{\mathbf{k}}-\mathbf{v}_{\mathbf{i}}\right\|^{2}+\eta^{2} \sum_{i=1}^{C}\left\|\mathbf{v}_{\mathbf{i}}-\mathbf{t}_{\mathbf{i}}\right\|^{2}+\alpha^{2}\|\mathbf{C} \mathbf{u}\|^{2}\right\}
\end{aligned}
$$


The difference from the formulation of Teranishi et al. (2013) is that two smoothness regularization parameters $\alpha_{\mathrm{g}}$ and $\alpha_{\mathrm{m}}$ are integrated into one parameter $\alpha$ which controls weight of membership smoothness in the FCM clustering. To optimise regularization parameters $\alpha$ and $\beta$ in the FCM clustering, we applied the L-curve criterion (Arai, 2003) to the FCM clustering.

We remark that incorporating smoothness regularization into $\mathrm{FCM}$ clustering enables us to save inversion computation time considerably. That is the reason why we do not have to repeat inversion calculation as many as the number of regularization parameter $\alpha$ and $\beta$.

\section{TEST MODEL}

In order to test our joint inversion algorithm, we consider the SMS model shown in Figure 1. The model consists of $17 \times 15 \times 12(3060)$ prisms in the analysis model $\left(340 \times 300 \times 96 \mathrm{~m}^{3}\right)$. Based on Evans (1996) and Tivey et al. (2002), we supposed the difference in density between SMS and surrounding volcanic rock is $2.5 \mathrm{~g} / \mathrm{cm}^{3}$ and that of magnetization is $\pm 4.0 \mathrm{~A} / \mathrm{m}$. We calculate anomalous data on the surface over a $18 \times 16$ grid with $20 \mathrm{~m}$ spacing in both directions. Since we assume that the measurement is conducted by remotely operated vehicle (ROV), $0.1 \mathrm{mGal}$ and $10 \mathrm{nT}$ Gaussian noise is added to the synthetic data. The observation level is assumed to be $1 \mathrm{~m}$ above the seabed, and the background magnetic field has constant inclination and declinations; $I=45^{\circ}, D=0^{\circ}$. The same inclination and declination are assigned for the magnetization vectors of rocks.

\section{EXAMPLES WITH SYNTHETIC DATA}

Figure 2 shows the cross-sections of true gravity and magnetization structure. Figures 3 and 4 show the results of four cross-sections of density and magnetization structure obtained from separate inversion using gravity data, separate inversion using magnetic data and joint inversion. Now, the similarity regularization parameter $\lambda$ is fixed to 10 . Throughout this paper, we assume that the total number of clusters, $\mathrm{C}$, is known $(\mathrm{C}=3)$.

In the both separated gravity and magnetic inversion results, the estimated models do not clearly indicate the locations and the stepwise shapes of assumed anomalous blocks. On the other hand, those from our joint inversion results show good agreement with the true model, especially around boundary of the stepwise structure. Joint inversion results determine density and

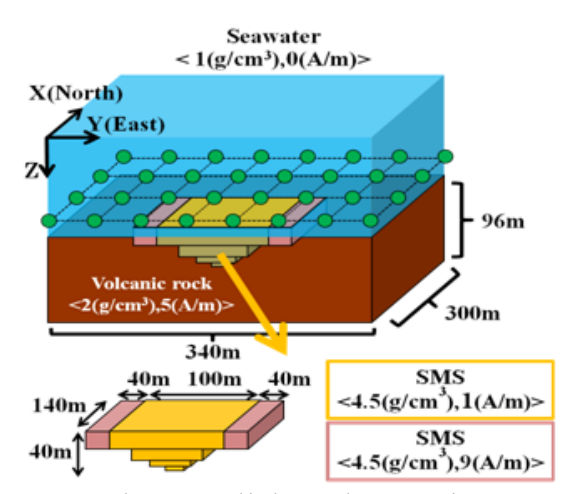

Figure 1. The parallel and resupinate pyramid SMS models. Density and magnetization of seawater, surrounding volcanic rock and SMS are described in the Figure 1.
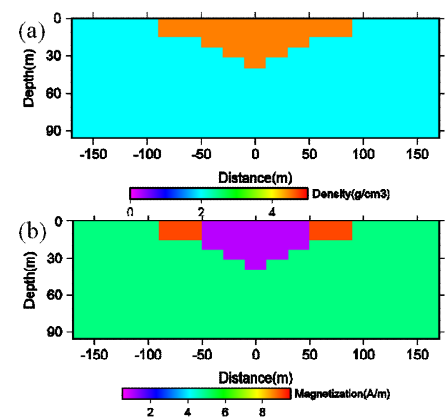

Figure 2. The true density (a) and magnetization (b) model. The vertical axis indicates depth below the seabed and the cross axis indicates horizontal distance. Each color scale illustrates amplitude of model parameter.
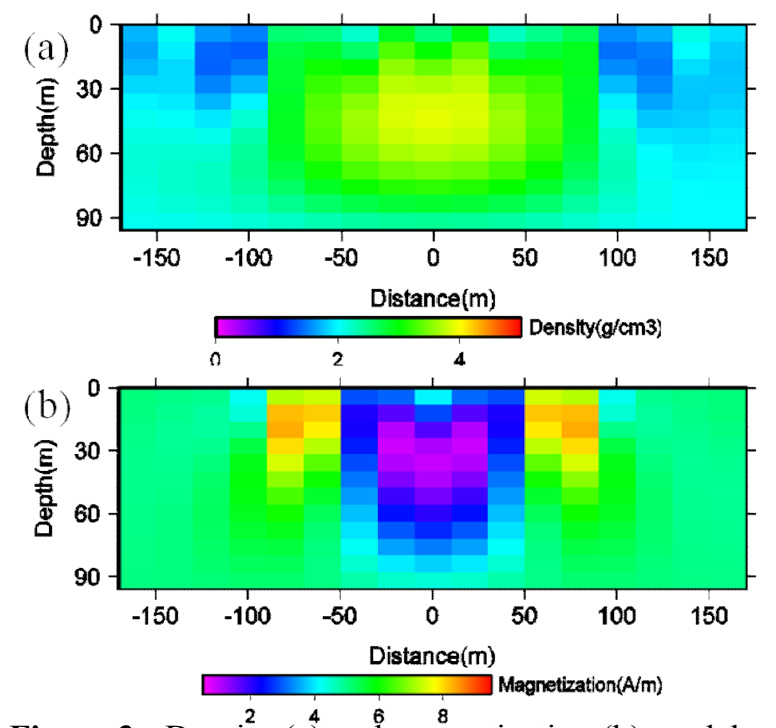

Figure 3. Density (a) and magnetization (b) model obtained by individual gravity and magnetic inversion respectively.

magnetization amplitude values better than the separated cases, and the shape of anomalies inferred from the synthetic data is close to the true models shown in Figure 2. Figure 5 shows distributions of density and magnetization values obtained by the gravity and magnetic inversions and joint inversion. 

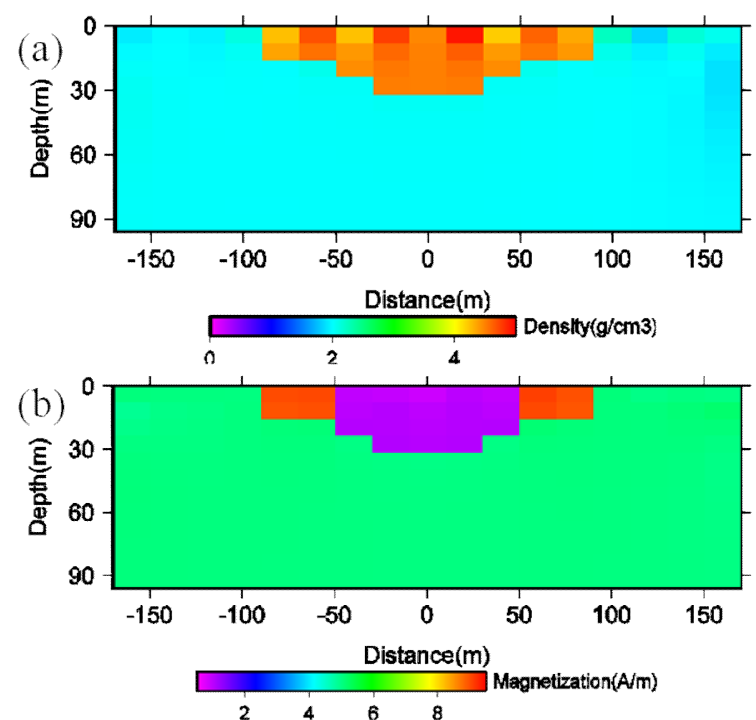

Figure 4. Density (a) and magnetization (b) model obtained by joint inversion.
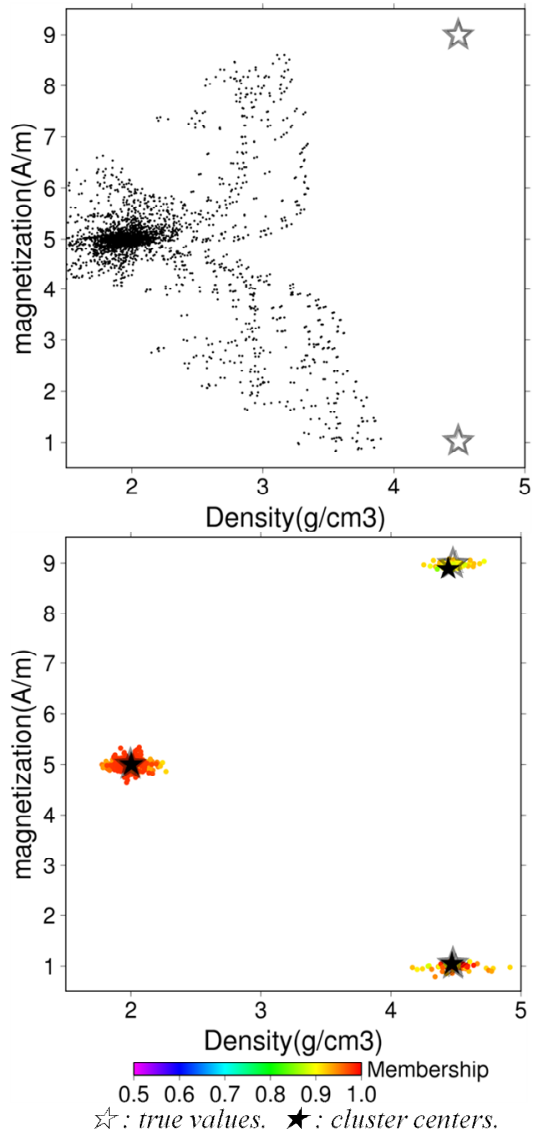

Figure 5. (top) The distribution of density and magnetization values obtained by the gravity and magnetic inversions. The white stars show the distribution of true density and magnetization values, (bottom) that of joint inversions and membership value at each cluster. The white stars show the distribution of true density and magnetization values. The block stars show the distribution of density and magnetization values of each cluster center.

\section{CONCLUSION}

We developed an algorithm to jointly invert two geophysical data sets using a constraint of FCM clustering between the density and magnetization. We calculated the noise-contaminated synthetic data to express a model of SMS deposit. In the individual inversion results, the resolution and accuracy of inverted results were not good though the results could detect underground anomalies of density and magnetization. On the other hand, in our joint inversion results, the inverted results showed better resolution and accuracy, and might partially overcome the inherent lack of depth resolution in gravity and magnetic data.

\section{REFERENCES}

1) Arai, E., 2003, Inversion of TDEM data for a horizontally layered earth using an L-curve criterion. BUTSURI-TANSA, 56, 117-130.

2) Bosch, M., 1999, Lithologic tomography: From plural geophysical data to lithology estimation. Journal of Geophysical Research, 104, 749-766.

3) Evans, R. L., 1996, A seafloor gravity profile across the TAG hydrothermal mound. Geophysical Research Letters, 23, 3447-3450.

4) Fregoso, E., and Gallardo, L. A., 2009, Cross-gradients joint 3D inversion with applications to gravity and magnetic data. Geophysics, 74, 31-42, doi:10.1190/1.3119263.

5) Hathaway, R. J., and Bezdek, J. C., 2001, Fuzzy c-means clustering of incomplete data. IEEE Transactions on Systems, Man, and Cybernetics, Part B: Cybernetics, 31, $735-744$.

6) Jegen, M.D., Hobbs, R.W., Tarits, P. \& Chave, A., 2009, Joint inversion of marine magnetotelluric and gravity data incorporating seismic con-straints. Preliminary results of sub-basalt imaging off the Faroe Shelf, Earth planet. Sci. Lett., 282, 47-55.

7) Sun, J., and Li, Y., 2012, Joint inversion of multiple geophysical data: A petrophysical approach using guided fuzzy c- means clustering, SEG Expanded Abs, doi:10.1190/segam20121388.1.

8) Li, Y., and Oldenburg, D.W., 1996, 3-D inversion of magnetic data. Geophysics, 61, 394-408.

9) Li, Y., and Oldenburg, D.W., 1998, 3-D inversion of gravity data. Geophysics, 63, 109-119.

10) Teranishi, Y., Mikada, H., Goto, T. and Takekawa, J., 2013, Three-dimensional joint inversion of gravity and magnetic anomalies using fuzzy c-means clustering, $75^{\text {th }}$ EAGE Conference \& Exhibition incorporating SPE EUROPEC 2013 London.

11) Tivey, M. A., and Johnson, H. P., 2002, Crustal magnetization reveals subsurface structure of Juan de Fuca Ridge hydrothermal vent fields. Geological Society of America, 30, 979-982. 